\title{
T-transformation Method for Studing the Multi-solitone Solutions of the Korteveg-de Vries Type Equations
}

\author{
Yuriy Turbal, Mariana Turbal, Andriy Bomba and Sokh Anastasiia \\ Department of Applied Mathematics, National University of Water Management and Nature Resourses Use, Rivne, 33022, Ukraine.
}

Received: September 19, 2014 / Accepted: October 24, 2014 / Published: July 25, 2015.

\begin{abstract}
In this paper, we propose a new technique of finding the PDE's traveling wave solutions based on the T-transformations. Using T-representation method we find a new class of Korteveg-de Vries solution and propose method for studing the multi-solitone solutions of the Korteveg-de Vries type equations .
\end{abstract}

Key words: Soliton; N-solitone solutions; Korteveg-de Vries equations; separated waves

\section{Introduction}

In resent years the investigation of separated waves plays an important role in many applied scientific fields. Travelling wave solutions can describe various phenomena in fluid mechanics, hydrodynamics, optics, plasma physics, solid state physics, biology , meteorology and other fields. Note that separated waves often occur at the boundaries of dynamic environments with different physical characteristics, such as " water-air " (in this case we consider the shallow water equations ), the limits of stratified fluids, on the verge of "gas- vacuum" (in [4] is considered thin gas disks that rotate in a gravitational field), on the border of crust and mantle (Moho surface ).

There are many models proposed to describe the physical phenomena of separated waves existence and variety of methods has been proposed to construct the exact and approximate solutions to nonlinear equations. It is well known the $\mathrm{KdV}$ equations which describes the uni-directional propagation of shallow-water waves and a number of generalizations, for example:

$$
u_{t}+\left(\alpha+\beta u^{p}\right) u^{p} u_{x}+u_{x x x}=0
$$

(is given by [10]),

Corresponding author: Yuriy Turbal, Ph.D., research field: mathematic modelling. E-mail: turbaly@gmail.com.

$$
u_{t}+a u^{2} u_{x}+b u_{x} u_{x x}+c u u_{x x x}+d u_{x x x x x}=0
$$

( is given by [11]),

$$
u_{t}=u_{x x x x x}+10 u u_{x x x}+20 u_{x} u_{x x x}+30 u^{2} u_{x}
$$

(is given by [12])

$$
u_{t}+u u_{x}+u_{x x x}=-\alpha u_{x x}-\beta u_{x x x x}
$$

(equation Kuramoto - Sivashynsky - KdV , [12]),

$$
u_{t}+u u_{x}+u_{x x x}-u_{x x x x x}=0
$$

(the Kavahara equation, [14]),

$$
\begin{gathered}
u_{t}+v u u_{x}+\mu u_{x x x}+\alpha u_{x x}+\gamma u_{x x x x}=0 \\
u_{t}+u u_{x}+\alpha u_{x x}+\beta u_{x x x}+u_{x x x x}=0
\end{gathered}
$$

(KdV - Burgers - Kuramoto [9]) and so on.

The KdV equations extended to several physical problems such as long internal waves in a density stratified ocean, acoustic waves on a crystal lattice and so on. In recent years many efficient methods of finding the traveling wave solutions was developed such as Infinite Series method [1], Backlund transformation method [2], Darboux transformation [3], tanh method [4, 5] extended tanh function method [6], modified and extended tanh function method [7], the 
generalized hyperbolic function [8], the variable separation method, first integral method, exp-function method [9] and so on .

In this paper, we propose a new technique of finding the PDE's traveling wave solutions which is based on the T-transformations.

\section{New Solution of KdV Equation Based on the T-representation}

Let $\tilde{x}(t)$ is function, which determine the point of maximum wave disturbance. Define a function that describe the shape of the wave in the form $\exp (-g(x-\widetilde{x}(t)))$, where $g($.$) is sufficiently smooth$ function, which satisfy conditions:

$$
g(x) \geq 0, g(0)=0, g^{(i)}(0)=0, i=\overline{1,3}
$$

Obviously that function $\gamma \exp (-g(x-\tilde{x}(t)) / \varepsilon)$ can describe a positive perturbation of any shape $(\gamma$ is amplitude parameter, $\varepsilon$ is a parameter that determines the location of disturbance). In the simplest case function $g($.$) is measure that defined on the set of$ intervals in $R^{1}$.

Let $\gamma$ be a function, $\gamma=\gamma(t)$. Hence we consider the solution we are looking for in the form:

$$
u(x, t)=\gamma(t) \exp (-g(x-\tilde{x}(t)) / \varepsilon)
$$

It is easy to build some kind of solution (2) generalization for the system of differential equations in partial derivatives:

The representation (2) make possible to find new aproach to ivestigate the multisolitone solution. In the case of small or large parameter $\varepsilon$ we can investigate some infinitesimal properties of travelling wave solution. Consider an example, the well-known Korteweg -de Vries equation in the form :

$$
u_{t}+6 u u_{x}+u_{x x x}=0
$$

We can write the derivatives of the function $u(x, t)$ :

$$
\begin{gathered}
u_{x}=-\frac{g^{\prime}(x-\tilde{x}(t))}{\varepsilon} u=\eta u ; \\
u_{x x}=\eta_{x} u+\eta u_{x}=\eta_{x} u+\eta^{2} u=\left(\eta_{x}+\eta^{2}\right) u ;
\end{gathered}
$$

$$
\begin{aligned}
& u_{x x x}=\left(\eta_{x x}+2 \eta \eta_{x}\right) u+\left(\eta_{x}+\eta^{2}\right) \eta u \\
& =\left(\eta_{x x}+3 \eta \eta_{x}+\eta^{3}\right) u ; \\
& u_{t}=\left(\gamma^{\prime}(t) / \gamma(t)+\frac{g^{\prime}(x-\widetilde{x}(t))}{\varepsilon} \widetilde{x}^{\prime}(t)\right) u \\
& =\left(\gamma^{\prime}(t) / \gamma(t)-\eta \widetilde{x}^{\prime}(t)\right) u .
\end{aligned}
$$

Substituting derivatives into equation (3) we obtain the equation:

$$
\begin{gathered}
\left(\gamma^{\prime}(t) / \gamma(t)-\eta \tilde{x}^{\prime}(t)\right) u+ \\
6 u \eta u+\left(\eta_{x x}+3 \eta \eta_{x}+\eta^{3}\right) u=0
\end{gathered}
$$

Let $x-\tilde{x}(t)=0$. Then, based on the properties of functions $g($.$) from the equation (7) we obtain:$

$$
\gamma^{\prime}(t) / \gamma(t) u=0 .
$$

Therefore we can formulate proposition:

Proposition 0. All travelling wave solutions of the $K d V$ equation have constant amplitude .

From the equation (3) we obtain:

$$
-\eta \widetilde{x}^{\prime}(t) u+6 u \eta u+\left(\eta_{x x}+3 \eta \eta_{x}+\eta^{3}\right) u=0
$$

Equation (5) define a general condition for functions $g(.) \in G$ and $\tilde{x}(t)$ at representation (3).

Formulate the following statement.

Proposition 1. Infinitely wide soliton (2) $(\lim u(x, t)=\gamma)$ is a trivial solution of the $K d V$ $\varepsilon \rightarrow \infty$ equation.

This property follows from the analysis of the boundaries of the form:

$$
\begin{aligned}
& \lim _{\varepsilon \rightarrow \infty}\left(\frac{g^{\prime}(x-\widetilde{x}(t))}{\varepsilon} \widetilde{x}_{t}(t)-6 \frac{g^{\prime}(x-\widetilde{x}(t))}{\varepsilon} u+\right. \\
& \left(-\frac{g^{\prime \prime \prime}(x-\widetilde{x}(t))}{\varepsilon}+3 \frac{g^{\prime \prime}(x-\widetilde{x}(t))}{\varepsilon} \frac{g^{\prime}(x-\widetilde{x}(t))}{\varepsilon}+\right. \\
& \frac{g^{\prime}(x-\widetilde{x}(x, t))}{\varepsilon} \widetilde{x}_{x x x}(x, t)- \\
& \left.\left(\frac{g^{\prime}(x-\widetilde{x}(x, t))}{\varepsilon}\right)^{3}\left(1-\widetilde{x}_{x}(x, t)\right)^{3}\right) u=0
\end{aligned}
$$

Proposition 2. Infinitely narrow soliton (2) $\left(\lim _{\varepsilon \rightarrow 0} u(x, t)=0\right)$ is a solution of the $K d V$. 
This proposition folows from the limits:

$$
\begin{aligned}
& \lim _{\varepsilon \rightarrow 0+}\left(\frac{g^{\prime}(x-\tilde{x}(t))}{\varepsilon} \widetilde{x}_{t}(t)-6 \frac{g^{\prime}(x-\tilde{x}(t))}{\varepsilon} u\right) u+ \\
& \left(-\frac{g^{\prime \prime \prime}(x-\tilde{x}(t))}{\varepsilon}+3 \frac{g^{\prime \prime}(x-\tilde{x}(t))}{\varepsilon} \frac{g^{\prime}(x-\tilde{x}(t))}{\varepsilon}-\right. \\
& \left.\left(\frac{g^{\prime}(x-\tilde{x}(t))}{\varepsilon}\right)^{3}\right) u=0 .
\end{aligned}
$$

It is obviously that similar properties have the solutions of other equations KdV-type that admit a trivial solution. At the same time, special $\mathrm{KdV}$ with variable coefficients species

$$
u_{t}+\alpha(t) u u_{x}+k \alpha(t) u_{x x x}=F(t) \quad(\text { is given by }[15])
$$

does not have the appropriate properties.

Let $\widetilde{x}(x, t)=v t$. Then (5) can be expressed in the form:

$$
\begin{gathered}
\frac{g^{\prime}(x-v t)}{\varepsilon} v-6 \frac{g^{\prime}(x-v t)}{\varepsilon} \gamma e^{-\frac{g(x-v t)}{\varepsilon}}+\left(-\frac{g^{\prime \prime \prime}(x-v t)}{\varepsilon}+\right. \\
\left.3 \frac{g^{\prime \prime}(x-v t)}{\varepsilon} \frac{g^{\prime}(x-v t)}{\varepsilon}-\left(\frac{g^{\prime}(x-v t)}{\varepsilon}\right)^{3}\right)=0 .
\end{gathered}
$$

Let $g(.) / \varepsilon=\tilde{g}(),. x-v t=y$. Then we obtain:

$$
\begin{aligned}
& \widetilde{g}^{\prime \prime \prime}(y)-3 \widetilde{g}^{\prime \prime}(y) \widetilde{g}^{\prime}(y)+\left(\widetilde{g}^{\prime}(y)\right)^{3}-v \widetilde{g}^{\prime}(y) . \\
& +6 \gamma \widetilde{g}^{\prime}(y) e^{-g(y)}=0
\end{aligned}
$$

Let $\tilde{g}^{\prime}(y)=p(g)$. Than

$$
\begin{gathered}
\widetilde{g}^{\prime \prime}(y)=p p^{\prime} ; \tilde{g}^{\prime \prime \prime}(y)=\left(p^{\prime \prime} p+\left(p^{\prime}\right)^{2}\right) p \\
\left(p^{\prime \prime} p+\left(p^{\prime}\right)^{2}\right)-3 p p^{\prime}+(p)^{2}-v+6 \gamma e^{-g}=0
\end{gathered}
$$

Let: $w=p^{2}$. Therefore:

$$
\begin{gathered}
w^{\prime}=2 p p^{\prime} ; w^{\prime \prime}=2\left(p^{\prime}\right)^{2}+2 p p^{\prime \prime} ; \\
w^{\prime \prime \prime}=4 p^{\prime} p^{\prime \prime}+2 p^{\prime} p^{\prime \prime}+2 p p^{\prime \prime \prime}=6 p^{\prime} p^{\prime \prime}+2 p p^{\prime \prime \prime} ; \\
w^{\prime \prime} / 2-3 w^{\prime} / 2+w=v-6 \psi e^{-\widetilde{g}} .
\end{gathered}
$$

We obtain linear equation. The partial solution can be obtained in the form:

$$
\begin{aligned}
& \quad w=k+l e^{-g} \cdot \text { Than } \\
& l e^{-g} / 2+3 l e^{-g} / 2+k+l e^{-\widetilde{g}}=v-6 \psi e^{-\widetilde{g}}, k=v, \\
& l=-2 \psi . \\
& \text { The general solution is: } \\
& \quad w=v-2 \psi e^{-\widetilde{g}}+C_{1} e^{2 \widetilde{g}}+C_{2} e^{\widetilde{g}} .
\end{aligned}
$$

Therefore $\quad p= \pm \sqrt{v-2 \psi e^{-\widetilde{g}}+C_{1} e^{2 \widetilde{g}}+C_{2} e^{\tilde{g}}}$, $\tilde{g}^{\prime}(y)= \pm \sqrt{v-2 \psi e^{-\widetilde{g}}+C_{1} e^{2 \widetilde{g}}+C_{2} e^{\widetilde{g}}}$.

We obtain the condition:

$$
\begin{gathered}
v-2 \psi e^{-\widetilde{g}}+C_{1} e^{2 \widetilde{g}}+C_{2} e^{\widetilde{g}} \geq 0, \\
v-2 \psi+C_{1}+C_{2}=0 .
\end{gathered}
$$

Under the condition of (6), we have $C_{1} \geq 0$.

Finally we can define the function $\tilde{g}(y)$ as solving of Koshi problem:

$$
\begin{gathered}
\widetilde{g}^{\prime}(y)=\operatorname{sign}(y) \sqrt{v-2 \psi e^{-\widetilde{g}}+C_{1} e^{2 \widetilde{g}}+C_{2} e^{\widetilde{g}}}, \\
\tilde{g}(0)=0 .
\end{gathered}
$$

The well known solution of $\mathrm{KdV}$ equation can be shown in the form :

$$
u(x, t)=2 \chi^{2} \operatorname{ch}^{-2} \chi\left(x-4 \chi^{2} t-\varphi\right),
$$

where $\chi, \varphi$ are parameters.

Let us prove that (8) is a partial case of (2). Let $C_{1}=C_{2}=0$. Then from (6) we obtain: $v=2 \psi$. Problem (7) can be shown in the form:

$$
g^{\prime}(y)=\sqrt{v-v e^{-g}}, g(0)=0 .
$$

Taking into account (8) and (2), we get :

$$
\begin{gathered}
2 \chi^{2} \operatorname{ch}^{-2}\left(\chi\left(x-4 \chi^{2} t-\varphi\right)\right)= \\
v \exp (-g(x-v t-\varphi)) / 2
\end{gathered}
$$

Therefore: $g(x-v t-\varphi)=2 \ln \operatorname{ch}(\chi(x-v t-\varphi))$. 
Let: $x-v t-\varphi=y$. Then:

$$
g(y)=2 \ln \operatorname{ch}(x y)
$$

Let $v=4 \chi^{2}$. It is easy to prove that (10) is solving of (9). Differenciating $g(y)$ we obtain : $g^{\prime}(y)=\frac{2 \chi \operatorname{sh}(\chi y)}{\operatorname{ch}(\chi y)}$. Substituting (10) into right side of (9), we get:

$$
\begin{aligned}
& \sqrt{v-v e^{-g}}=2 \chi \sqrt{1-e^{-2 \ln c h(\chi y)}} \\
& =2 \chi \sqrt{1-\operatorname{ch}^{-2}(\chi y)}= \\
& =2 \chi \sqrt{\left(\operatorname{ch}^{2}(\chi y)-1\right) / \operatorname{ch}^{2}(\chi y)} \\
& =2 \chi \sqrt{\operatorname{sh}^{2}(\chi y) / \operatorname{ch}^{2}(\chi y)}= \\
& =2 \chi \operatorname{sh}(\chi y) / \operatorname{ch}(\chi y)=g^{\prime}(y)
\end{aligned}
$$

The proof is completed.

\section{Method for Studing the Interaction of Solitons}

Let $\quad u_{i}(x, t)=\gamma_{i} \exp \left(-g_{i}\left(x-\tilde{x}_{i}(t)\right) / \varepsilon_{i}\right), i=1,2 \quad-$ exact solutions of the KdV equation. Obviously, the function $u(x, t)=u_{1}(x, t)+u_{2}(x, t)$ is not a solution of the $\mathrm{KdV}$ equation because of its nonlinearity. Therefore, we consider the following generalization.

Let $u_{1}(x, t)=\gamma_{1}(t) \exp \left(-g_{1}\left(x-\tilde{x}_{1}(t)-\alpha\right) / \varepsilon_{1}\right)$, $u_{2}(x, t)=\gamma_{2}(t) \exp \left(-g_{2}\left(x-\tilde{x}_{2}(t)-\beta\right) / \varepsilon_{1}\right) \quad$ where, $\alpha, \beta$ some parameters. We introduce some effective radii soliton localization $R_{\delta}^{1}, R_{\delta}^{2}$ :

$$
\begin{aligned}
& R_{\delta}^{1}=\min \left\{R:\left|u_{1}(x, t)\right|<\delta,\left|x-\tilde{x}_{1}(t)\right|>R\right\} \\
& R_{\delta}^{2}=\min \left\{R:\left|u_{2}(x, t)\right|<\delta,\left|x-\tilde{x}_{2}(t)\right|>R\right\}
\end{aligned}
$$

Denote $G_{\delta}^{1}(t)=\left\{x:\left|x-\tilde{x}_{1}(t)\right|<R_{\delta}^{1}\right\}$,

$$
G_{\delta}^{2}(t)=\left\{x:\left|x-\widetilde{x}_{2}(t)\right|<R_{\delta}^{2}\right\} .
$$

It is obvious that the function $u(x, t)$ satisfies the $\mathrm{KdV}$ equation in a domain $\left\{G_{\delta}^{1} \cap \bar{G}_{\delta}^{2} \cup \bar{G}_{\delta}^{1} \cap G_{\delta}^{2} \cup \bar{G}_{\delta}^{1} \cap \bar{G}_{\delta}^{2}\right\}$ with accuracy $O(\delta)$ if $u_{i}(x, t)$ are accurate solutions of the $\mathrm{KdV}$ equation. Moreover, in view of assertion 0 , the condition should be: $\gamma_{i}(t)=\gamma_{i}=$ const if $\left|\widetilde{x}_{2}(t)-\widetilde{x}_{1}(t)\right|>R_{\delta}^{1}+R_{\delta}^{2}$.

Let $\tilde{x}_{i}^{\prime}(t)=c_{i} \gamma_{i}(t), c_{i}=2, i=1,2$.Substituting the sum $u_{1}(x, t)+u_{2}(x, t)$ into equation (6) for the case $x=\tilde{x}_{1}(t)+\alpha$ we obtain:

$$
\begin{aligned}
& \gamma_{1}{ }^{\prime}(t) \exp \left(-g_{1}(\alpha) / \varepsilon_{1}\right)+\gamma_{2}{ }^{\prime}(t) \exp \left(-g_{2}(-d(t)) / \varepsilon_{2}\right) \\
& +g_{1}{ }^{\prime}(\alpha) \tilde{x}_{1}{ }^{\prime}(t) \tilde{u}_{1}(\alpha, t) / \varepsilon_{1}+g_{2}{ }^{\prime}(-d(t)) \tilde{x}_{2}{ }^{\prime}(t) \gamma_{2}(t) \\
& \times \exp \left(-g_{2}(-d(t)) / \varepsilon_{2}\right) / \varepsilon_{2}+6\left(\gamma_{1}(t) \exp \left(-g_{1}(\alpha) / \varepsilon_{1}\right)\right. \\
& \left.+\gamma_{2}(t) \exp \left(-g_{2}(-d(t)) / \varepsilon_{2}\right)\right)\left(-g_{1}{ }^{\prime}(\alpha) \tilde{u}_{1}(\alpha, t) / \varepsilon_{1}\right. \\
& \left.-g_{2}{ }^{\prime}(-d(t)) u_{2}(-d(t), t) / \varepsilon_{2}\right)+\left(-g_{1}{ }^{\prime \prime}(\alpha) / \varepsilon_{1}\right. \\
& \left.+3 g_{1}{ }^{\prime}(\alpha) g_{1}{ }^{\prime}(\alpha) / \varepsilon_{1}{ }^{2}-\left(g_{1}{ }^{\prime}(\alpha)\right)^{3} / \varepsilon_{1}{ }^{3}\right) \tilde{u}_{1}(\alpha, t) \\
& +\left(-g_{2}{ }^{\prime \prime}(-d(t)) / \varepsilon_{2}+3 g_{2}{ }^{\prime}(-d(t)) g_{2}{ }^{\prime \prime}(-d(t)) / \varepsilon_{2}{ }^{2}\right. \\
& \left.-\left(g_{2}{ }^{\prime}(-d(t))\right)^{3} / \varepsilon_{2}{ }^{3}\right) \gamma_{2}(t) \exp \left(-g_{2}(-d(t)) / \varepsilon_{2}\right)=0 \\
& \text { where } d(t)=\widetilde{x}_{2}(t)-\widetilde{x}_{1}(t)-\alpha . \\
& \text { Similarly in the case of } x=\widetilde{x}_{2}(t)+\beta, \\
& d_{1}(t)=\tilde{x}_{2}(t)+\beta-\tilde{x}_{1}(t):
\end{aligned}
$$

$\gamma_{1}{ }^{\prime}(t) \exp \left(-g_{1}\left(d_{1}(t)\right) / \varepsilon_{1}\right)+\gamma_{2}{ }^{\prime}(t) \exp \left(-g_{2}(\beta) / \varepsilon_{2}\right)$

$+g_{1}{ }^{\prime}\left(d_{1}(t)\right) \tilde{x}_{1}{ }^{\prime}(t) u_{1}\left(d_{1}(t), t\right) / \varepsilon_{1}$

$+g_{2}{ }^{\prime}(\beta) \tilde{x}_{2}{ }^{\prime}(t) u_{2}(\beta, t) / \varepsilon_{2}+6\left(\gamma_{1}(t) \exp \left(-g_{1}\left(d_{1}\right.\right.\right.$

$\left.\left.(t)) / \varepsilon_{1}\right)+\gamma_{2}(t) \exp \left(-g_{2}(\beta) / \varepsilon_{2}\right)\right)\left(\left(-g_{1}{ }^{\prime}\left(d_{1}\right.\right.\right.$

$\left.(t)) \gamma_{1}(t) \exp \left(-g_{1}\left(d_{1}(t)\right) / \varepsilon_{1}\right) / \varepsilon_{1}\right)$

$\left.-g_{2}{ }^{\prime}(\beta) \tilde{u}_{2}(\beta, t) / \varepsilon_{2}\right)+\left(\left(-g_{2}{ }^{\prime \prime}(\beta) / \varepsilon_{2}\right.\right.$

$\left.\left.+3 g_{2}{ }^{\prime}(\beta) g^{\prime \prime}(\beta) / \varepsilon_{2}^{2}-\left(g_{2}{ }^{\prime}(\beta)\right)^{3} / \varepsilon_{2}{ }^{3}\right)\right) \tilde{u}_{2}(\beta, t)$

$+\left(-g_{1}{ }^{\prime \prime}\left(d_{1}(t)\right) / \varepsilon_{1}+3 g_{1}{ }^{\prime}\left(d_{1}(t)\right) g_{1}{ }^{\prime \prime}\left(d_{1}(t)\right) / \varepsilon_{1}{ }^{2}-\right.$

$\left.\left(g_{1}{ }^{\prime}\left(d_{1}(t)\right)\right)^{3} / \varepsilon_{1}^{3}\right) \gamma_{1}(t) \exp \left(-g_{1}\left(d_{1}(t)\right) / \varepsilon_{1}\right)=0$.

Hence we obtain the system of equations: 


$$
\begin{aligned}
& \tilde{x}_{1}^{\prime}(t)=2 \gamma_{1}(t), \tilde{x}_{2}^{\prime}(t)=2 \gamma_{2}(t), \\
& \gamma_{1}{ }^{\prime}(t)=-\exp \left(-g_{2}(\beta) / \varepsilon_{2}\right)\left(g_{1}{ }^{\prime}(\alpha) \tilde{x}_{1}{ }^{\prime}(t) \tilde{u}_{1}(\alpha, t) / \varepsilon_{1}+g_{2}{ }^{\prime}(-d(t)) \tilde{x}_{2}{ }^{\prime}(t) \gamma_{2}(t) \exp \left(-g_{2}(-d(t)) / \varepsilon_{2}\right) / \varepsilon_{2}\right. \\
& +6\left(\gamma_{1}(t) \exp \left(-g_{1}(\alpha) / \varepsilon_{1}\right)+\gamma_{2}(t) \exp \left(-g_{2}(-d(t)) / \varepsilon_{2}\right)\right)\left(-g_{1}{ }^{\prime}(\alpha) \tilde{u}_{1}(\alpha, t) / \varepsilon_{1}-g_{2}{ }^{\prime}(-d(t)) \tilde{u}_{2}(-d(t), t) / \varepsilon_{2}\right) \\
& +\left(-g_{1}{ }^{\prime \prime}(\alpha) / \varepsilon_{1}+3 g_{1}{ }^{\prime}(\alpha) g_{1}{ }^{\prime \prime}(\alpha) / \varepsilon_{1}{ }^{2}-\left(g_{1}{ }^{\prime}(\alpha)\right)^{3} / \varepsilon_{1}^{3}\right) \tilde{u}_{1}(\alpha, t)+\left(-g_{2}{ }^{\prime \prime}(-d(t)) / \varepsilon_{2}+3 g_{2}{ }^{\prime}(-d(t)) g_{2}{ }^{\prime \prime}\right. \\
& \left.\left.(-d(t)) / \varepsilon_{2}{ }^{2}-\left(g_{2}{ }^{\prime}(-d(t))\right)^{3} / \varepsilon_{2}^{3}\right) \gamma_{2}(t) \exp \left(-g_{2}(-d(t)) / \varepsilon_{2}\right)\right)+\left(g_{1}{ }^{\prime}\left(d_{1}(t)\right) \tilde{x}_{1}{ }^{\prime}(t) \gamma_{1}(t) \exp \left(-g_{1}\left(d_{1}(t)\right) / \varepsilon_{1}\right) / \varepsilon_{1}\right. \\
& +g_{2}{ }^{\prime}(\beta) \tilde{x}_{2}{ }^{\prime}(t) \tilde{u}_{2}(\beta, t) / \varepsilon_{2}+6\left(\gamma_{1}(t) \exp \left(-g_{1}\left(d_{1}(t)\right) / \varepsilon_{1}\right)+\gamma_{2}(t) \exp \left(-g_{2}(\beta) / \varepsilon_{2}\right)\right)\left(\left(-g_{1}{ }^{\prime}\left(d_{1}(t)\right) \gamma_{1}(t)\right.\right. \\
& \left.\left.\exp \left(-g_{1}\left(d_{1}(t)\right) / \varepsilon_{1}\right) / \varepsilon_{1}\right)-g_{2}{ }^{\prime}(\beta) \tilde{u}_{2}(\beta, t) / \varepsilon_{2}\right)+\left(\left(-g_{2}{ }^{\prime \prime}(\beta) / \varepsilon_{2}+3 g_{2}{ }^{\prime}(\beta) g^{\prime \prime}(\beta) / \varepsilon_{2}{ }^{2}-\left(g_{2}{ }^{\prime}(\beta)\right)^{3} / \varepsilon_{2}{ }^{3}\right)\right) \tilde{u}_{2}(\beta, t) \\
& \left.\left.\left.+\left(-g_{1}{ }^{\prime \prime}\left(d_{1}(t)\right) / \varepsilon_{1}+3 g_{1}{ }^{\prime}\left(d_{1}(t)\right) g_{1}{ }^{\prime \prime}\left(d_{1}(t)\right) / \varepsilon_{1}{ }^{2}-\left(g_{1}{ }^{\prime}\left(d_{1}(t)\right)\right)^{3} / \varepsilon_{1}^{3}\right) \tilde{u}_{1}\left(d_{1}(t), t\right) / \varepsilon_{1}\right)\right) \exp \left(-g_{2}(-d(t)) / \varepsilon_{2}\right)\right) / \\
& \left(\exp \left(-g_{1}(\alpha) / \varepsilon_{1}-g_{2}(\beta) / \varepsilon_{2}\right)-\exp \left(-g_{1}(d(t)) / \varepsilon_{1}-g_{2}\left(-d_{1}(t)\right) / \varepsilon_{2}\right),\right. \\
& \gamma_{2}{ }^{\prime}(t)=-\exp \left(-g_{1}(\alpha) / \varepsilon_{1}\right)\left(g_{1}{ }^{\prime}\left(d_{1}(t)\right) \tilde{x}_{1}{ }^{\prime}(t) \gamma_{1}(t) \exp \left(-g_{1}\left(d_{1}(t)\right) / \varepsilon_{1}\right) / \varepsilon_{1}+g_{2}{ }^{\prime}(\beta) \tilde{x}_{2}{ }^{\prime}(t) u_{2} / \varepsilon_{2}\right. \\
& +6\left(\gamma_{1}(t) \exp \left(-g_{1}\left(d_{1}(t)\right) / \varepsilon_{1}\right)+\gamma_{2}(t) \exp \left(-g_{2}(\beta) / \varepsilon_{2}\right)\right)\left(\left(-g_{1}{ }^{\prime}\left(d_{1}(t)\right) \gamma_{1}(t) \exp \left(-g_{1}\left(d_{1}(t)\right) / \varepsilon_{1}\right) / \varepsilon_{1}\right)\right. \\
& \left.-g_{2}{ }^{\prime}(\beta) u_{2} / \varepsilon_{2}\right)+\left(\left(-g_{2}{ }^{\prime \prime}(\beta) / \varepsilon_{2}+3 g_{2}{ }^{\prime}(\beta) g^{\prime \prime}(\beta) / \varepsilon_{2}{ }^{2}-\left(g_{2}{ }^{\prime}(\beta)\right)^{3} / \varepsilon_{2}{ }^{3}\right)\right) u_{2} \\
& \left.+\left(-g_{1}{ }^{\prime \prime}\left(d_{1}(t)\right) / \varepsilon_{1}+3 g_{1}{ }^{\prime}\left(d_{1}(t)\right) g_{1}{ }^{\prime \prime}\left(d_{1}(t)\right) / \varepsilon_{1}^{2}-\left(g_{1}{ }^{\prime}\left(d_{1}(t)\right)\right)^{3} / \varepsilon_{1}^{3}\right) \gamma_{1}(t) \exp \left(-g_{1}\left(d_{1}(t)\right) / \varepsilon_{1}\right)\right) \\
& +\exp \left(-g_{1}(d(t)) / \varepsilon_{1}\right)\left(g_{1}{ }^{\prime}(\alpha) \tilde{x}_{1}{ }^{\prime}(t) u_{1} / \varepsilon_{1}+g_{2}{ }^{\prime}(-d(t)) \tilde{x}_{2}{ }^{\prime}(t) \gamma_{2}(t) \exp \left(-g_{2}(-d(t)) / \varepsilon_{2}\right) / \varepsilon_{2}\right. \\
& +6\left(\gamma_{1}(t) \exp \left(-g_{1}(\alpha) / \varepsilon_{1}\right)+\gamma_{2}(t) \exp \left(-g_{2}(-d(t)) / \varepsilon_{2}\right)\right)\left(-g_{1}{ }^{\prime}(\alpha) u_{1} / \varepsilon_{1}-g_{2}{ }^{\prime}(-d(t)) u_{2} / \varepsilon_{2}\right) \\
& +\left(-g_{1}{ }^{\prime \prime}(\alpha) / \varepsilon_{1}+3 g_{1}{ }^{\prime}(\alpha) g_{1}{ }^{\prime \prime}(\alpha) / \varepsilon_{1}{ }^{2}-\left(g_{1}{ }^{\prime}(\alpha)\right)^{3} / \varepsilon_{1}^{3}\right) u_{1}+\left(-g_{2}{ }^{\prime \prime}(-d(t)) / \varepsilon_{2}+3 g_{2}{ }^{\prime}(-d(t)) g_{2}{ }^{\prime \prime}(-d(t)) / \varepsilon_{2}{ }^{2}\right. \\
& \left.\left.-\left(g_{2}{ }^{\prime}(-d(t))\right)^{3} / \varepsilon_{2}{ }^{3}\right) \gamma_{2}(t) \exp \left(-g_{2}(-d(t)) / \varepsilon_{2}\right)\right) /\left(\exp \left(-g_{1}(\alpha) / \varepsilon_{1}-g_{2}(\beta) / \varepsilon_{2}\right)-\exp \left(-g_{1}(d(t)) / \varepsilon_{1}-g_{2}\left(-d_{1}(t)\right) / \varepsilon_{2}\right)\right. \text {. }
\end{aligned}
$$

Writing the obvious initial conditions :

$$
\begin{aligned}
& \gamma_{1}(0)=\gamma_{1}^{0} \exp \left(-g_{1}(\alpha) / \varepsilon_{1}\right), \\
& \gamma_{2}(0)=\gamma_{2}^{0} \exp \left(-g_{2}(\beta) / \varepsilon_{2}\right),
\end{aligned}
$$

we get new Cauchy problem and can build the exact solution in the area:

$$
\Omega_{\alpha \beta}=\left\{(x, t): x=\tilde{x}_{1}(t)+\alpha, x=\tilde{x}_{2}(t)+\beta\right\} .
$$

Similarly, we can consider and different laws of wave motion. The N-solitone solutions for any $N>2$ can be always constructed in a similar way.

\section{Numerical Results}

Let consider some numerical results for special case of the functions $g(y)$ and $u(x, t)$. Consider an example of solitone interaction. According to approach given in chapter 3, define the function $g_{1}(y), g_{2}(y)$. Let

$$
\begin{gathered}
g_{1}(y)=2 \ln (\cosh (\sqrt{v 1 / 4} y)), \\
g_{2}(y)=2 \ln (\cosh (\sqrt{v 2 / 4} y)), \\
v 1=0.3, v 2=0.1 .
\end{gathered}
$$

Solving the problem (13) with initial condition

$$
\begin{aligned}
& \gamma_{1}(0)=0.15, \gamma_{2}(0)=0.05, \\
& \tilde{x}_{1}(0)=0, \tilde{x}_{2}(0)=20
\end{aligned}
$$

we can construct function $u_{1}(x, t)+u_{2}(x, t)$ (Fig.1). 


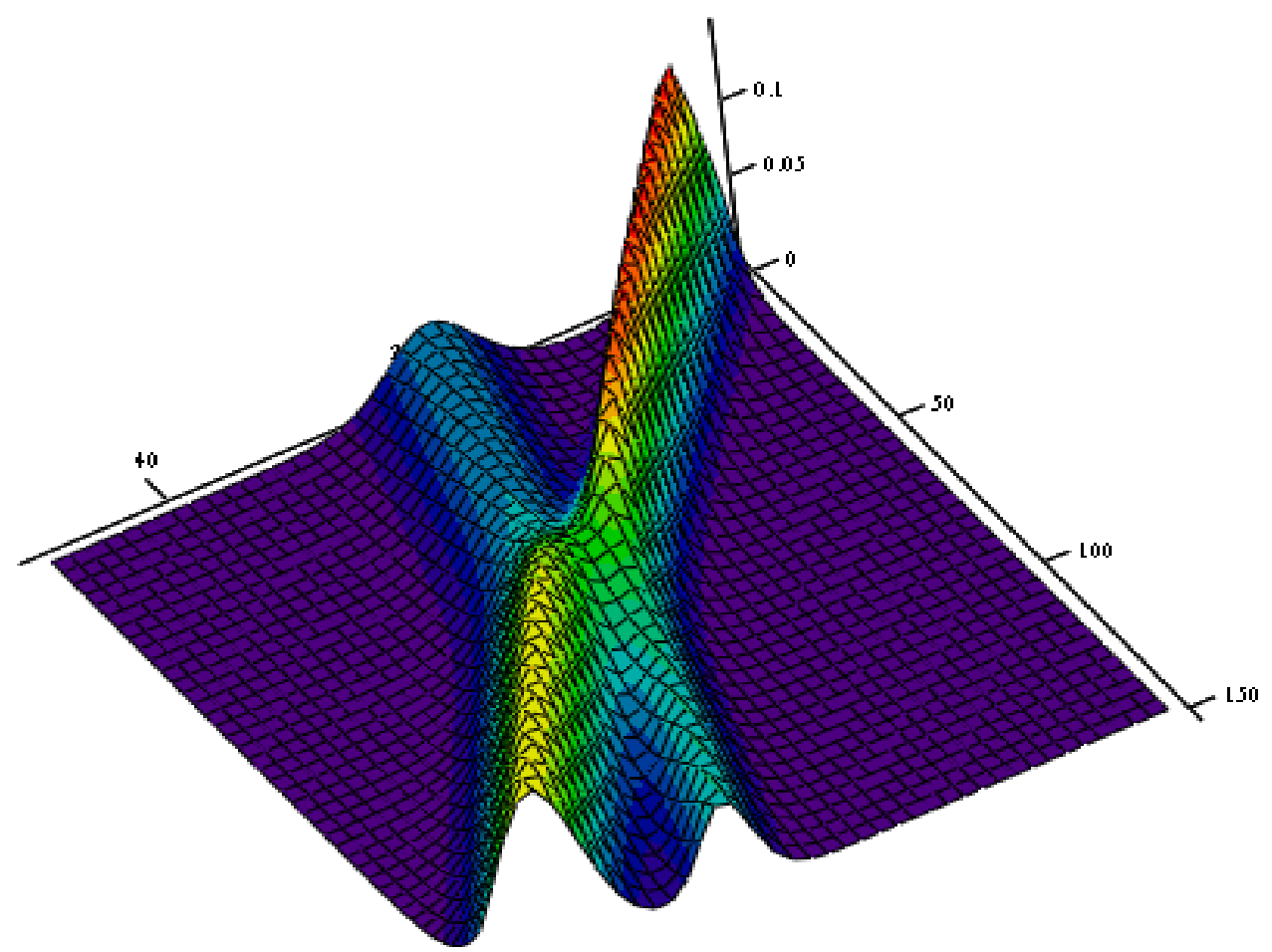

Fig.1 The 3D grafs of two-solitone solutions.

\section{Conclusions}

In this paper, we propose a new technique of finding the PDE's traveling wave solutions; this technique is based on the T-transformations. Using $\mathrm{T}$-representation method we find a new class of $\mathrm{KdV}$ solution and proove that well known solution (10) is a partial case of representation (3).

The proposed method can be applied to finding solutions of other differential equations in partial derivatives in the form of solitary waves. Also this method can be useful for the investigation multi-solitone solutions.

\section{References}

[1] A.Asaraii Infinite Series Method for Solving the Improved Modified KdV Equation, Studies in Mathematical Sciences, Vol.4, No.2,2012,pp.25-31

[2] Miura, M. R.(1978). Backlund Transformation. Berlin: Springer-Verlag , 4

[3] Matveev, V. B. \& Salle, M. A. (1991). Darboux Transformations and Solitons. Berlin: Heideelberg Springer

[4] Parkes, E. J. \& Duffy, B. R. (1998). An Automated Tanh-Function Method for Finding Solitary Wave
Solution to Nonlinear Evolution Equations. Comput. Phys. Commun., 98, 288-300.

[5] Evans, D. J. \& Raslan, K. R. (2005). The Tanh Function Method for Solving Some Important Non-Linear Partial Differential Equation. Int. J. comput. Math., 82, 897-905.

[6] Fan E. (2000). Extended Tanh-Function Method and Its Applications to Nonlinear Equations. Phys. Lett. A., 277, 212-218

[7] Elwakil, S. A., EL-Labany, S. K., Zahran, S. K. \& Sabry, M. A. (2002). Modified Extended Tanh-Function Method For Solving Nonlinear Partial Differential Equations. Phys. Lett. A., 299, 179-188 2-118.

[8] Gao, Y. T. \& Tian, B. (2001). Generalized

[9] Hyperbolic- Function Method with Computerized Symbolic Computation to Construct the Solitonic Solutions to Nonlinear Equations of Mathematical Physics Comput. Phys. Commun., 133, 158-164c.

[10] Jae-Myouhg Kim and Changbum Chun, New Exact Solutons to the Kdv-Burgers-Kuramoto Equation whith the Exp-Function Method, Abstract and Applied Analysis, Volume 2012, Article ID 892420.

[11] E.V. Krishnan and Q.J. A. Khan, Higher-order KdV-Type Equations and their stability, IJMMS 27:4 (2001) 215-220

[12] G.Adomian, The fifth-order korteveg-de vries equation, Internet J. Math. \& Math. Sci., Vol. 19, No. 2 (1996) 415

[13] Maomao Cai, Dening Li, and Chontita Rattanakul The Coupled Kuramoto Sivashinsky-KDV Equations for 


\section{Korteveg-de Vries Type Equations}

Surface Wave in Multilayed Liquid Films Mathematical Physics, Volume 2013, Article ID 673546

[14] Maomao Cai, Dening Li, and Chontita Rattanakul The Coupled Kuramoto Sivashinsky-KDV Equations for Surface Wave in Multilayed Liquid Films Mathematical Physics, Volume 2013, Article ID 673546

[15] Gleb G. Doronin \& Nikolay A. Larkin Well and ill-posed problems for the $\mathrm{KdV}$ and Kawahara equations, Bol. Soc. Paran. Mat. (3s.) v.26 1-2 (2008):133-137

[16] Alvaro H. Salas and Cesar A.Gomez S, Exact Solutions for a Third-Order $\mathrm{KdV}$ Equation whith Variable Coefficients and Forcing Term, Mathematical Problems in Engineering, Volume 2009, article ID 737928 